\title{
Plumbing for Liquid Chromatography
}

\author{
Lane C. Sander \\ National Institute of Standards and Technology, \\ Gaithersburg, MD 20899, USA \\ lane.sander@nist.gov
}

Video DOI: http://doi.org/10.18434/T4DK5T

Key words: fluidic components; high pressure connections; instrumentation; liquid chromatography.

Accepted: December 1, 2016

Published: January 12, 2017

https://doi.org/10.6028/jres.122.006

\section{Summary}

The setup and maintenance of liquid chromatographic equipment requires a basic knowledge of chromatographic hardware. Tubing connections are an essential component in liquid chromatography instrumentation. System function and performance depend on the selection and proper installation of tubing and end fittings. Problems with these components can result in solvent leaks, degraded peak shape, and system failure. This presentation will provide information about the various kinds of chromatographic fittings and related components that are in common use, and will demonstrate how to form high pressure connections for stainless steel and PEEK tubing. Numerous close-up photographs are included to illustrate subtle differences in existing parts, and video examples show proper ways to cut and connect tubing. ${ }^{1}$

\footnotetext{
${ }^{1}$ Contribution of the National Institute of Standards and Technology. Not subject to copyright. Certain commercial equipment, instruments, or materials are identified to specify adequately the experimental procedure. Such identification does not imply recommendation or endorsement by the National Institute of Standards and Technology, nor does it imply that the materials or equipment identified are the best available for the purpose.
} 